Bara S Minwah BDS, MSc, FICBS (Lec.)

Mohmmed K Hassouni BDS, FDSRCRS (Lec.)

\section{Evaluation of Naproxen and Ibuprofen efficacy on post maxillomandibular fixation trismus}

\section{Department Oral and Maxillofacial surgery} College of Dentistry, University of Mosul

Department Oral and Maxillofacial surgery College of Dentistry, University of Mosul

الخلاصة

يعد كسر الفلك السفلي الأكثر شيو عا في حالات الجروح الرضية للوجه ،ورهنة هنالك طريقتان لعلاج كسر الفك السفلي هما: ،الطريقة

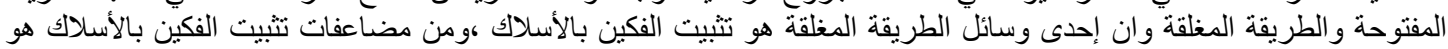

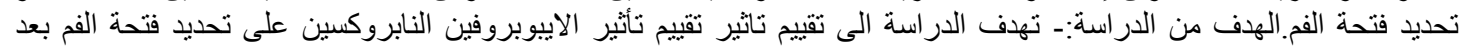

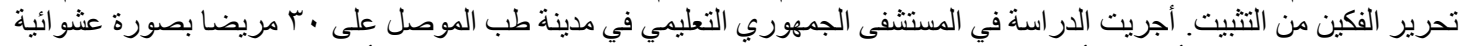

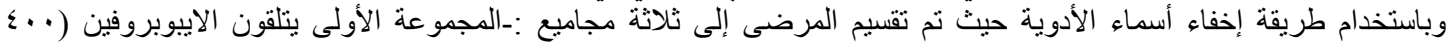

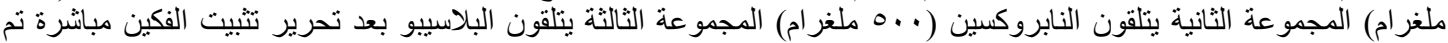

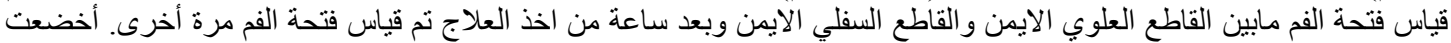

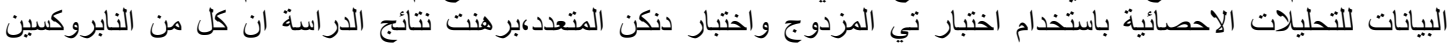

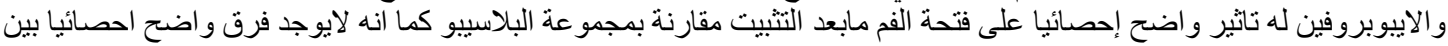

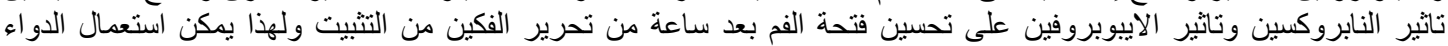

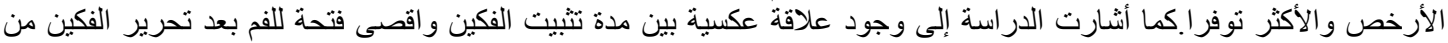

\title{
ABSTRACT
}

Aims of the study: The study aims to evaluate the effect of naproxen and ibuprofen on post maxillomandibular fixation (MMF) limited mouth opening. Materials and Methods: The study was performed at AL-Jumhoori Teaching Hospital in Mosul City on 30 patients with mandibular fracture and lor maxilla who were treated with MMF. The medicaments were allocated to 30 patients using a double blind randomization study who were divided into three groups .Group 1(10 patients) received Ibuprofen. Group 2(10 patients) received Naproxen and Group 3 (10 patients) received placebo . Immediately after MMF release, the maximum mouth opening was measured as the inter-incisal distance between the right upper central incisor and the right lower central incisor. After one hour ,the inter incisal distance was measured again and recorded .The data were analyzed using paired T-test and Duncan multiple analysis. Results: Both Naproxen and Ibuprofen have a statistically significant effect on the improvement of post-MMF limited mouth opening compared to placebo. At the same time there was no significant difference between the effect of Naproxen and that of Ibuprofen on the improvement of post MMF-limited mouth opening . The study also showed that there is inverse correlation between the MMF period and the maximum mouth opening immediately after MMF release. Conclusions: There is no significant difference between the effect of naproxen and that of ibuprofen on the improvement of limited mouth opening after MMF release. The available and cheaper drugs can be prescribed.

Key words: Fracture mandible, MMF, Trismus, Naproxen and Ibuprofen.

Minwah BS, Hassouni MYM. Evaluation of Naproxen and Ibuprofen efficacy on post maxillomandibular fixation trismus. Al-Rafidain Dent J. 2011; 11(2): 376-380.

Received: 2/6/2010 Sent to Referees: $21 / 6 / 2010$

Accepted for Publication: 27/7/2010

\section{INTRODUCTION}

The mandible is reportedly the most commonly fractured bone in facial trauma (1). The shape of the mandible and the presence of weak areas, as well as being movable and exposed to direct trauma have all made it more vulnerable to fracture in comparison to mid-face fracture ${ }^{(2)}$. The etiology of mandibular fracture varies according to the area in which the survey is taken. The common causes of man- dibular fracture include vehicle accidents, falls from height, sports injury and war injury ${ }^{(3)}$.Treatment of mandibular fracture can be divided into open and close techniques, one of the closed methods is maxillomandibular fixation (MMF) which is formerly known as (IMF).The mandible must be immobilized for 4-6 weeks for most types of fractures ${ }^{(1)}$. Immobilization of the jaw for long periods may lead to muscle spasm and limited mouth opening. 
Normal mouth opening in healthy adults is reported to be $36-46 \mathrm{~mm}$. When a measured inter-incisal opening is less than $35 \mathrm{~mm}$, it means there is a limited mouth opening ${ }^{(4)}$. Because of the limited mouth opening ,diet may be restricted ,speech and appearance also impaired and behavioral deficit may arise ${ }^{(5)}$. Trismus tends to develop slowly. In some patients, it cannot be noticed until they can only open $20 \mathrm{~mm}$ or less. Treatment that begins early in the condition is likely to be more effective ${ }^{(6)}$.

Over the years ,many methods have been attempted to treat limited mouth opening. An example of these methods is wooden tongue spatula ,acrylic screw and trans-cutaneous electrical nerve stimulation (TENS). The most important cause of trismus is pain which can be alleviated by the use of NSAIDs.This study was carried out to evaluate the effects of two types of NSAIDs which are naproxen and ibuprofen on post MMF Trismus.

\section{MATERIALS AND METHODS}

Patients: The study comprised thirty patients who were treated for mandibular fracture by closed reduction and fixation with MMF (maxillomandibular fixation) which was formerly named as IMF or inter maxillary fixation. Their jaws were immobilized for a period of 3-6 weeks. They were randomly selected and divided into 3 groups of 10 patients for each group. The patients included in this study were clinically healthy and had no contra-indication for the administration of NSAIDs. Patients with traumatized anterior teeth were excluded from this study.

Grouping: Three groups of capsules were prepared, one group contains $400 \mathrm{mg}$ ibuprofen, the $2^{\text {nd }}$ group contains $500 \mathrm{mg}$ naproxen, while the $3^{\text {rd }}$ group contained sugar. Each group of capsule was given a code of either A, B or C. These were prepared and coded by the pharmacist. Neither the examiner nor the patient knew the contents of any capsule when it was administered by the patients (double blind selection).Patients were randomly selected into 3 groups of 10 patients for each group. In group 1, the MMF was released and the patient ingested a capsule coded A. In group 2, the MMF was released and the patient ingested a capsule coded B. In group 3, the MMF was released and the patient ingested a capsule coded $\mathrm{C}$.

Exercise: After release of the MMF and capsule ingestion, each patient started on jaw exercise for 60 minutes with the aid of a wooden tongue spatula to overcome trismus due to muscle spasm. The simplest method of doing the exercises was by the insertion of a wooden tongue spatula between the upper and lower dental arches, with the number of spatulas increased gradually.

Mouth Opening Measurement: After releasing the MMF, the patient was asked to open hislher mouth as widely as possible until pain was felt and the distance was measured from the incisal edge of the right upper central incisor to the incisal edge of the right lower central incisor using a dental vernier "Munchner Model.". The maximum inter incisal distance was measured and recorded at minute 1 and at minute 60 following capsule ingestion using the dental vernier "Munchner Model".

\section{RESULTS}

In the ibuprofen treated group, there was a significant difference between mouth opening at minute one after MMF removal and that at minute 60 as shown in Table (1) and as shown in Figure (1).

Table (1): Effect of Ibuprofen, Naproxen and Placebo on mouth opening Using paired t-test.

\begin{tabular}{llll}
\hline Drug & $\begin{array}{l}\text { Mean } \pm \text { SD } \\
\text { Opening at 1 min }\end{array}$ & Opening at 60 min & $p$-value \\
\hline Ibuprofen & $13.50 \pm 5.44$ & $23.40 \pm 5.27$ & $<0.001$ \\
Naproxen & $17.10 \pm 6.86$ & $27.60 \pm 4.62$ & $<0.001$ \\
Placebo & $20.30 \pm 7.01$ & $20.60 \pm 6.93$ & $0.08 \mathrm{NS}$ \\
\hline
\end{tabular}




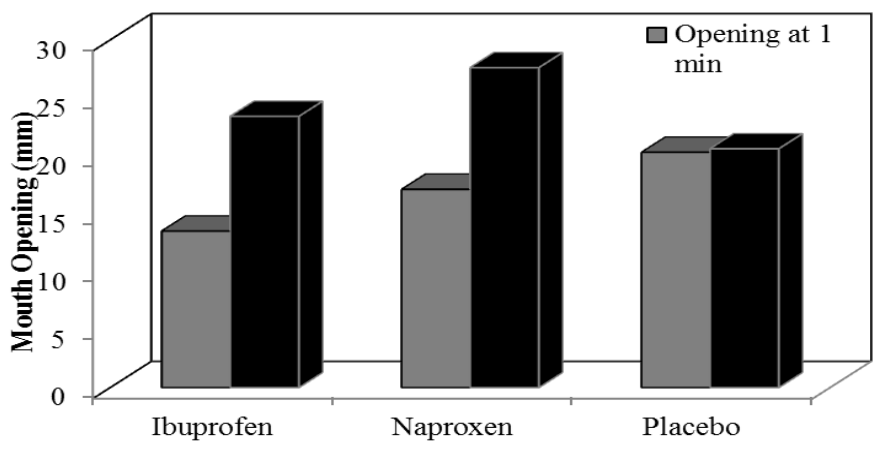

Figure 1: Effect of Ibuprofen, Naproxen and Placebo on mouth opening

In the naproxen treated group,there was a significant difference between mouth opening at minute one after MMF removal and that at minute 60 as shown in Table (1) and as shown in Figure (1). In the placebo treated group ,there is no significant difference between mouth opening at minute one after MMF removal and that at minute 60 as shown in Table (1)and as shown in Figure (1).

The improvement among the three groups were accounted as : The improvement $=(($ Mouth opening at $60 \mathrm{~min}-$ mouth opening at 1 minute ) Mouth opening at minute one ) $\times 100$

There was no significant difference in the improvement of mouth opening achieved in the brufen treated group and that of naproxen treated group ,while, there was a significant difference in the improvement of mouth opening achieved by the ibuprofen treated group and that of the placebo treated group, and, there was a significant difference in the improvement of mouth opening and that of placebo treated group ,as shown in Table (2).

Table (2): Comparison of mouth opening improvement after 60 minutes of operation among drug.

\begin{tabular}{ccc}
\hline Drugs & $\begin{array}{c}\text { Percent Improvement After 60 Minutes } \\
\text { Mean } \pm \text { SE }\end{array}$ & Duncan Group \\
\hline Ibuprofen & $100.63 \pm 33.19$ & $\mathrm{~A}$ \\
Naproxen & $92.59 \pm 34.06$ & $\mathrm{~A}$ \\
Placebo & $1.67 \pm 0.86$ & $\mathrm{~B}$ \\
\hline
\end{tabular}

Different letters vertically mean significant difference at $p \leq 0.05$ using paired t-test.

Comparing the number of improved cases: There was a significant difference between the number of improved cases in the naproxen treated group and ibuprofen treated group and that of placebo treated group . (BTG:10, NTG :10,PTG:3)

Correlation : There was significant inverse correlation between the duration of MMF period and the mouth opening at minute 1 Figure(2).

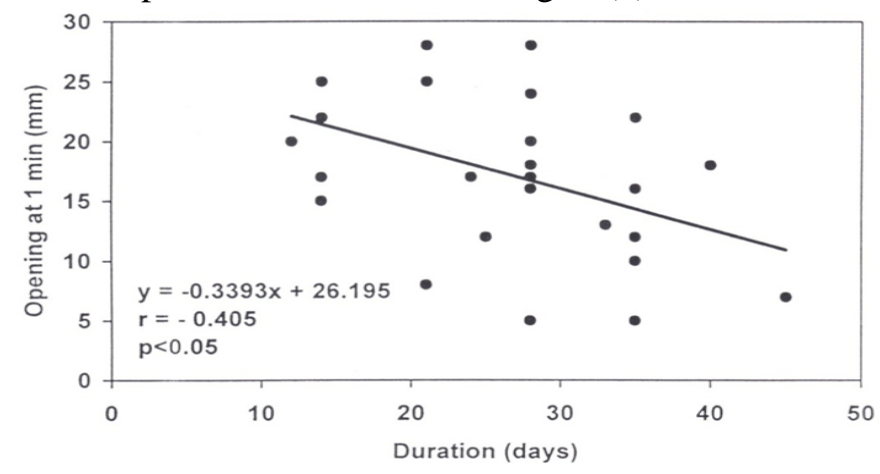

Figure (2): Relationship between duration of fixation and opening at minute 1 


\section{DISCUSSION}

In the ibuprofen treated group (BTG),there was a significant difference between mouth opening at minute one and that at minute 60 after MMF removal .All the ten cases within the group showed improvement in mouth opening. Several clinical studies have been performed on the efficacy of ibuprofen in controlling post operative trismus. Alkaskus (7) showed that both ibuprofen and paracetamol have a significant effect on post traumatic limited mouth opening.

In the naproxen treated group ,there was a significant difference in mouth opening at minute one and that at minute 60 after MMF removal. All ten cases within the group showed improvement in mouth opening. Allen and Bobbie concluded that the administration of naproxen sodium in the immediate post operative period may be indicated for optimum post operative analgesia ${ }^{(8)}$.Kirsch et al showed a significant superiority in pain reduction of naproxen than paracetamol ${ }^{(9)}$.

In the placebo treated group there were no significant differences between mouth opening at minute one and that at minute 60 after MMF removal and in three of ten cases showed improvement in mouth opening. Placebos are pharmacologically inert substances that nonetheless have therapeutic effects .They act by alleviating anxiety and are fairly effective in a high percentage of cases. It has been suggested that placebo analgesia may be mediated by endorphins( naturally occurring morphine) like pain reducing substances ${ }^{(10)}$.

There was a significant inverse correlation between the duration of MMF period and mouth opening at minute one after MMF removal. Fouad (11) concluded that a short period of MMF (14 days ) followed by an arch bar wired to the lower jaw is a suitable alternative to conventional MMF period (28 days ) for the treatment of the fracture of the mandibular teeth bearing area in that there is a significant difference in the degree of mouth opening limitation.

\section{CONCLUSIONS}

Most of the patients with mandibular fractures who are treated by maxillomandibular MMF, will complain from limited mouth opening after MMF release There is no significant difference between the effect of ibuprofen and that of naproxen on the improvement of limited mouth opening after MMF release, so the most cheaper drug can be prescribed.

A short period of MMF (14 days) followed by an arch bar wired to the lower jaw is a suitable alternative to conventional MMF (28 days )for the treatment of the fracture of the mandibular teeth bearing area.

\section{REFERENCES}

1. Anderson T and Alpert B. Experience with rigid fixation of mandibular fractures and immediate function . J Oral Maxillofacial Surg 1992;50: 555-560.

2. AL-Rawee RY. Incidence of maxillofacial trauma in Nineveh government. M,Sc, Thesis, University of Mosul ; 1997.

3. Dongas P, Hall GM. Mandibular fracture patterns in Tasmania , Australia. Aust Dent J. 2002;47(2):131-137.

4. Inchimura $\mathrm{K}$, Tanaka $\mathrm{T}$.Trismus in patients with malignant tumor $\mathrm{s}$ in the head and neck. J Laryngol Otol. 1993;107:1017-1018.

5. Kummona R. Functional rehabilitation of ankylosed temporomandibular joints. Oral surg Oral med Oral path .1978; 46 (4) : 495-505.

6. Ammaratunga NA. Mouth opening after release of maxillomandibular fixation in fractured patient. J Oral Maxillofacial Surg. 1987; 45(5): 383-385.

7. Alkaskus HH. The efficacy of ibuprofen and naproxen on post operative complaints following removal of impacted lower third molar ,M,Sc, Thesis. University of Mosul; 2001.

8. Allen $\mathrm{L}$ and Bobbie $\mathrm{J}$.Comparison of preoperative and post operative naproxen sodium for suppression of post operative pain Oral Maxillofacial Surg. 1990; 48(7): 674-678.

9. Kirsch TA , Halladay SC, Koschic M . A double blind randomized study of naproxen sodium, ibuprofen, and placebo in post operative dental pain. Clin Therap. 1993; 15 (5) : 845-854.

10.Epstein JB . Understanding placebo in dentistry. JADA 1984; 109: 71-72.

11.Fouad AA. Treatment of Traumatic Mandibular fracture of the mandibular 
